\title{
Device-based therapies for resistant hypertension in chronic kidney disease: The continuing quest for a cure
}

\section{Device-based therapies have shown promise, but} many questions still need clarification
$\mathrm{H}$ YPERTENSION can be a cause or consequence of chronic kidney disease (CKD), in which sodium retention and volume expansion, increased activity of the renin-angiotensin system, and increased sympathetic nervous system activity can all contribute to raising blood pressure. Resistant hypertension, which is associated with a higher risk for adverse outcomes, is overrepresented in CKD. An analysis from the CRIC (Chronic Renal Insufficiency Cohort) study showed a high prevalence of apparent treatment-resistant hypertension in CKD (about 40\%), with a 38\% increase in risk for adverse cardiovascular events and 28\% increase in risk for adverse renal events. ${ }^{1}$ This underscores the importance of blood pressure control in this population, and novel therapeutic strategies should be explored.

See related article, page 435

In this issue, Gajulapalli et $\mathrm{al}^{2}$ review device-based therapies (renal denervation, baroreflex activation and amplification therapy, arteriovenous coupling, and renal artery stenting) in the treatment of resistant hypertension in CKD.

While clinical trials of device-based therapies have shown promise, many questions still need clarification. Additionally, as noted in the article, most device trials excluded patients with moderate-to-severe CKD (stages $3 \mathrm{~b}, 4$, and 5) and patients with end-stage kidney disease. In analyzing these trials, some important considerations need to be kept in mind.

doi:10.3949/ccjm.87a.20092

\section{RENAL DENERVATION}

The concept of renal denervation is arguably the most exciting new frontier for devicebased therapies.

However, in resistant hypertension, the large SYMPLICITY HTN-3 renal denervation trial did not show benefit in a group undergoing this therapy compared with a sham procedure group. ${ }^{3}$ Methodologic concerns with this study and questions related to ablation technique led to more-refined trial designs.

The SPYRAL HTN-ON MED 4 and OFF$\mathrm{MED}^{5}$ trials used second-generation multielectrode radiofrequency ablation devices, and the RADIANCE-HTN SOLO trial ${ }^{6}$ used an endovascular ultrasound device, all of which showed significant blood pressure reductions in the denervation group. It should be noted that the SPYRAL HTN-OFF MED and RADIANCE-HTN SOLO trials were done in patients not taking antihypertensive medications, and SPYRAL HTN-ON MED was done in patients taking up to 3 antihypertensive medications, which does not necessarily constitute resistant hypertension; the mean number of antihypertensive medications in the denervation group was 2.2.

Unblinded trials like DENERHTN in resistant hypertension showed significantly more blood pressure reduction when renal denervation was combined with stepped-up medication therapy, but there was no sham procedure group in this study.

Meta-analyses of randomized trials of renal denervation showed reductions in 24- 
hour ambulatory blood pressure, with a mean difference of $-4.02 /-2.05 \mathrm{~mm} \mathrm{Hg}$ compared with controls, and a mean difference of $-3.65 /-1.71 \mathrm{~mm} \mathrm{Hg}$ when the analysis was restricted to sham-controlled randomized trials, suggesting a modest effect size. ${ }^{8,9}$ No subgroup analysis for CKD was done in these trials.

There are limited data on renal denervation in CKD with resistant hypertension. A small study in 15 patients with stage 3 or 4 CKD (mean estimated glomerular filtration rate [eGFR] $31 \mathrm{~mL} / \mathrm{min} / 1.73 \mathrm{~m}^{2}$ ) showed significant reduction in office blood pressure and ambulatory nighttime blood pressure, but not in 24-hour ambulatory blood pressure or ambulatory daytime blood pressure. ${ }^{10}$ The REGINA RDN study in 25 patients with stage 3 or 4 CKD (mean eGFR $37 \mathrm{~mL} / \mathrm{min} / 1.73 \mathrm{~m}^{2}$ ) did not show a significant change in 24-hour ambulatory blood pressure, although there was significant reduction in office blood pressure. ${ }^{11}$

It is reassuring that available data do not suggest an unfavorable renal safety profile with denervation. A 3-year follow-up from the Global SYMPLICITY registry, which is a database of real-world patients treated with renal denervation, did not find a decline in CKD exceeding what would be expected in hypertensive CKD patients. ${ }^{12}$

Besides limited data in CKD patients, there are other unanswered questions with renal denervation:

Who are the optimal candidates? Response to renal denervation has been variable, and to date there are no good predictors of response that would enable optimal patient selection.

How much ablation is enough? There is no method to verify adequacy of ablation in real time.

Is the effect durable? Long-term efficacy (beyond 3 years) is as yet unknown. Could reinnervation occur, and what are the possible physiologic effects of this?

Is it safe in the long term? While the reported safety profile so far is reassuring, could there be longer-term deleterious vascular effects?

Will it improve cardiovascular outcomes? While cardiovascular outcomes could be extrapolated from effects of lower blood pressure, no studies of renal denervation have directly examined cardiovascular outcomes.

\section{BAROREFLEX AMPLIFICATION}

Baroreflex amplification therapy is the other device-based therapy of interest, with endovascular deployment of a self-expanding nitinol device in the carotid sinus that increases vessel radius and amplifies baroreceptor signaling. (An earlier concept used an electrical device to stimulate the baroreceptors.)

CALM-FIM_EUR, a proof-of-principle study, showed significant lowering of 24-hour ambulatory blood pressure, but patients with moderate to severe CKD were excluded from this study (the average eGFR was $83 \mathrm{~mL} /$ $\left.\min / 1.73 \mathrm{~m}^{2}\right) .{ }^{13}$ Results from the CALM 2 study are awaited, although this trial also excluded patients with eGFR less than $45 \mathrm{~mL}$ / $\mathrm{min} / 1.73 \mathrm{~m}^{2}$.

\section{ARTERIOVENOUS COUPLING}

The coupler device, which creates a central arteriovenous fistula connecting the distal iliac vein and artery to reduce arterial resistance and pressure, is no longer in development. ${ }^{14}$

In summary, device-based therapies for resistant hypertension hold promise, but more research is needed, particularly in patients with advanced CKD. These therapies remain investigational in the United States and are not currently approved by the US Food and Drug Administration for clinical use.

\section{RENAL ARTERY STENTING}

As Gajulapalli et al note, ${ }^{2}$ angioplasty with stenting for atherosclerotic renal artery stenosis remains a matter of controversy, and recent trials did not show differences in cardiovascular outcomes, mortality, or progression of CKD with stenting compared with medical therapy alone (although the CORAL trial showed a modestly greater blood pressure reduction in the stent group). ${ }^{15}$ In our practice, we consider stenting for hemodynamically significant renal artery stenosis only in certain circumstances, including resistant hypertension with blood pressure that remains uncontrolled despite optimal and intensive medication therapy. ${ }^{16}$

\section{HOW SHOULD RESISTANT HYPERTENSION BE MANAGED?}

The management of resistant hypertension
Renal denervation may be the most exciting new devicebased therapy 


\section{TABLE 1}

\section{Approach to treatment-resistant hypertension}

\section{Confirm the diagnosis}

Blood pressure should be measured in an out-of-office setting using either ambulatory monitoring or home blood pressure monitoring to confirm diagnosis and to ascertain possible white coat effect.

\section{Carefully review medications}

Review of medications should include over-the-counter medications such as nonsteroidal anti-inflammatory drugs and herbal medications, if any.

\section{Explore the possibility of nonadherence to medications}

Nonadherence may be cost- or side-effect-related or due to complexity of regimen and poor understanding of medications.

\section{Reinforce lifestyle modifications}

Guidelines for nonpharmacologic therapy from the American Heart Association and American College of Cardiology include a low-sodium diet, physical activity, weight management, and limited alcohol intake. Of note, although increased intake of dietary potassium is recommended for hypertension, this would not be feasible for patients with chronic kidney disease, who are prone to hyperkalemia.

\section{Assess for secondary causes of hypertension}

\section{Ensure optimal doses and combination of antihypertensive medications}

Use thiazide-like diuretics such as chlorthalidone instead of hydrochlorothiazide.

Use loop diuretics in states of volume overload or when the estimated glomerular filtration rate is less than $30 \mathrm{~mL} / \mathrm{min} / 1.73 \mathrm{~m}^{2}$.

Dual renin-angiotensin blockade with angiotensin-converting enzyme inhibitor and angiotensin II receptor blocker is not recommended for hypertension as it has not been shown to improve cardiovascular outcomes or blood pressure control, and increases risk for acute kidney injury.

Consider addition of spironolactone as a fourth-line agent; this may require use of potassium binding agents in patients to prevent hyperkalemia.

Further stepwise treatment could include addition of beta-blockers, alpha-blockers, centrally acting alpha agonists, and direct vasodilators.

Complex treatment regimens should take into account the possibility of increased side effects and risk of nonadherence, and care should be individualized, with close monitoring of renal function and electrolytes.

\section{Refer to a hypertension specialist}

should follow a stepwise approach (Table 1), and should prompt investigation for secondary causes, including possible renal artery stenosis.

\section{Spironolactone as a fourth-line agent \\ Spironolactone has been shown to be ben- eficial as a fourth-line agent in resistant hypertension. ${ }^{17}$ A small Spanish trial (DE- NERVHTA) found that spironolactone low- ered 24-hour ambulatory blood pressure more than did renal nerve denervation. ${ }^{18}$ The larger Czech PRAGUE-15 study showed similar 24-}

hour ambulatory blood pressure reduction at 6 months with renal denervation compared with medication therapy that included spironolactone, and numerically better blood pressure reduction at 1 and 2 years in the group that was able to tolerate and continue spironolactone. ${ }^{19}$

In $\mathrm{CKD}$, the use of spironolactone may be more likely to cause hyperkalemia, especially when combined with angiotensinconverting enzyme inhibitors or angiotensin II receptor blockers. Newer gastrointestinal cation exchanger potassium-binding agents 
have been shown to be effective in enabling patients with CKD and resistant hypertension to take spironolactone with less hyperkalemia. $^{20}$

\section{DEVICES WOULD BE ATTRACTIVE}

Complex treatment regimens increase the possibility of side effects and risk of nonadherence, and device-based therapies would seem an attractive option especially in these patients. While this is certainly an area of opportunity, available data do not support recommendations for device therapy in this group (yet).

Resistant hypertension in CKD presents a dual challenge for management, and randomized trials are needed in resistant hypertension across the CKD spectrum to better assess the efficacy of device-based therapies and comparative outcomes with antihypertensive drug combinations.

\section{REFERENCES}

1. Thomas G, Xie D, Chen HY, et al; CRIC Study Investigators. Prevalence and prognostic significance of apparent treatment resistant hypertension in chronic kidney disease: report from the Chronic Renal Insufficiency Cohort Study. Hypertension 2016; 67(2):387-396. doi:10.1161/HYPERTENSIONAHA.115.06487

2. Gajulapalli RD, Chahine J, Rader F, Aneja A. Procedures and devices to treat resistant hypertension in chronic kidney disease. Cleve Clin J Med 2020; 87(7):435-443. doi:10.3949/ccjm.87a.19099

3. Bhatt DL, Kandzari DE, O'Neill WW, et al; SYMPLICITY HTN-3 Investigators. A controlled trial of renal denervation for resistant hypertension. N Engl J Med 2014; 370(15):1393-1401. doi:10.1056/ NEJMoa1402670

4. Kandzari DE, Böhm M, Mahfoud F, et al; SPYRAL HTN-ON MED Trial Investigators. Effect of renal denervation on blood pressure in the presence of antihypertensive drugs: 6-month efficacy and safety results from the SPYRAL HTN-ON MED proof-of-concept randomised trial. Lancet 2018; 391(10137):2346-2355.

doi:10.1016/S0140-6736(18)30951-6

5. Townsend RR, Mahfoud F, Kandzari DE, et al; SPYRAL HTN-OFF MED Trial Investigators. Catheter-based renal denervation in patients with uncontrolled hypertension in the absence of antihypertensive medications (SPYRAL HTN-OFF MED): a randomised, sham-controlled, proof-of-concept trial. Lancet 2017; 390(10108):2160-2170. doi:10.1016/S0140-6736(17)32281-X

6. Azizi M, Schmieder RE, Mahfoud F, et al; RADIANCE-HTN Investigators. Endovascular ultrasound renal denervation to treat hypertension (RADIANCE-HTN SOLO): a multicentre, international, single-blind, randomised, sham-controlled trial. Lancet 2018; 391(10137):2335-2345. doi:10.1016/S0140-6736(18)31082-1

7. Azizi M, Sapoval M, Gosse P, et al; Renal Denervation for Hypertension (DENERHTN) investigators. Optimum and stepped care standardised antihypertensive treatment with or without renal denervation for resistant hypertension (DENERHTN): a multicentre, open-label, randomised controlled trial. Lancet 2015; 385(9981):1957-1965. doi:10.1016/S0140-6736(14)61942-5

8. Sardar P, Bhatt DL, Kirtane AJ, et al. Sham-controlled randomized trials of catheter-based renal denervation in patients with hypertension. J Am Coll Cardiol 2019; 73(13):1633-1642. doi:10.1016/j.jacc.2018.12.082

9. Cheng X, Zhang D, Luo S, Qin S. Effect of catheter based renal denervation on uncontrolled hypertension: a systematic review and meta-analysis. Mayo Clin Proc 2019; 94(9):1695-1706. doi:10.1016/j.mayocp.2019.07.005

10. Hering D, Mahfoud F, Walton AS, et al. Renal denervation in moderate to severe CKD. J Am Soc Nephrol 2012; 23(7):1250-1257.
doi:10.1681/ASN.2011111062

11. Prasad B, Berry W, Goyal K, Dehghani P, Townsend RR. Central blood pressure and pulse wave velocity changes post renal denervation in patients with stages 3 and 4 chronic kidney disease: the Regina RDN Study. Can J Kidney Health Dis 2019 Feb 13; 6:2054358119828388. doi:10.1177/2054358119828388

12. Mahfoud F, Böhm M, Schmieder R, et al. Effects of renal denervation on kidney function and long-term outcomes: 3-year follow-up from the Global SYMPLICITY Registry. Eur Heart J 2019; 40(42):34743482. doi:10.1093/eurheartj/ehz118

13. Spiering W, Williams B, Van der Heyden J, et al; CALM-FIM_EUR investigators. Endovascular baroreflex amplification for resistant hypertension: a safety and proof-of-principle clinical study. Lancet 2017; 390(10113):2655-2661. doi:10.1016/S0140-6736(17)32337-1

14. Peixoto $\mathrm{AJ}$. Is there a role for device therapies in resistant hypertension? The CON side. Kidney360 January 2020; 1(1):9-13. doi:10.34067/KID.0000742019

15. Cooper CJ, Murphy TP, Cutlip DE, et al; CORAL Investigators. Stenting and medical therapy for atherosclerotic renal-artery stenosis. $\mathrm{N}$ Engl J Med 2014; 370(1):13-22. doi:10.1056/NEJMoa1310753

16. Textor SC, Lerman LO. Reality and renovascular disease: when does renal artery stenosis warrant revascularization? Am J Kidney Dis 2014; 63(2):175-177. doi:10.1053/j.ajkd.2013.11.004

17. Williams B, MacDonald TM, Morant S, et al; British Hypertension Society's PATHWAY Studies Group. Spironolactone versus placebo, bisoprolol, and doxazosin to determine the optimal treatment for drug-resistant hypertension (PATHWAY-2): a randomised, double-blind, crossover trial. Lancet 2015; 386(10008):2059-2068. doi:10.1016/S0140-6736(15)00257-3

18. Oliveras A, Armario $P$, Clarà $A$, et al. Spironolactone versus sympathetic renal denervation to treat true resistant hypertension: results from the DENERVHTA study - a randomized controlled trial. J Hypertens 2016; 34(9):1863-1871. doi:10.1097/HJH.0000000000001025

19. Rosa J, Widimský $P$, Waldauf $P$, et al. Renal denervation in comparison with intensified pharmacotherapy in true resistant hypertension: 2-year outcomes of randomized PRAGUE-15 study. J Hypertens 2017; 35(5):1093-1099. doi:10.1097/HJH.0000000000001257

20. Agarwal R, Rossignol P, Romero A, et al. Patiromer versus placebo to enable spironolactone use in patients with resistant hypertension and chronic kidney disease (AMBER): a phase 2, randomised, double-blind, placebo-controlled trial. Lancet 2019; 394(10208):15401550. doi:10.1016/S0140-6736(19)32135-X

ADDRESS: George Thomas, MD, Department of Nephrology and Hypertension, Q7, Cleveland Clinic, 9500 Euclid Avenue, Cleveland, OH 44195; thomasg3@ccf.org 